\title{
Methylation of yeast ribosomal protein Rpl3 promotes translational elongation fidelity
}

\author{
QAIS AL-HADID, KEVIN ROY, GUILLAUME CHANFREAU, and STEVEN G. CLARKE \\ Department of Chemistry and Biochemistry and the Molecular Biology Institute, University of California, Los Angeles, Los Angeles, \\ California 90095, USA
}

\begin{abstract}
Rpl3, a highly conserved ribosomal protein, is methylated at histidine 243 by the Hpm1 methyltransferase in Saccharomyces cerevisiae. Histidine 243 lies close to the peptidyl transferase center in a functionally important region of Rpl3 designated as the basic thumb that coordinates the decoding, peptidyl transfer, and translocation steps of translation elongation. Hpm1 was recently implicated in ribosome biogenesis and translation. However, the biological role of methylation of its Rpl3 substrate has not been identified. Here we interrogate the role of Rpl3 methylation at $\mathbf{H} 243$ by investigating the functional impact of mutating this histidine residue to alanine (rp/3-H243A). Akin to $\mathrm{Hpm} 1$-deficient cells, $r p / 3-\mathrm{H} 243 \mathrm{~A}$ cells accumulate $35 \mathrm{~S}$ and 235 pre-rRNA precursors to a similar extent, confirming an important role for histidine methylation in pre-rRNA processing. In contrast, Hpm1-deficient cells but not $\mathrm{rp} / 3-\mathrm{H} 243 \mathrm{~A}$ mutants show perturbed levels of ribosomal subunits. We show that Hpm1 has multiple substrates in different subcellular fractions, suggesting that methylation of proteins other than Rpl3 may be important for controlling ribosomal subunit levels. Finally, translational fidelity assays demonstrate that like Hpm1-deficient cells, rp/3-H243A mutants have defects in translation elongation resulting in decreased translational accuracy. These data suggest that Rpl3 methylation at $\mathrm{H} 243$ is playing a significant role in translation elongation, likely via the basic thumb, but has little impact on ribosomal subunit levels. Hpm1 is therefore a multifunctional methyltransferase with independent roles in ribosome biogenesis and translation.
\end{abstract}

Keywords: translational fidelity; protein histidine methylation; ribosomal protein; ribosome biogenesis; translation elongation

\section{INTRODUCTION}

The ribosome is a nanomachine composed of RNA and proteins that work in concert with tRNAs and protein factors to translate the mRNA into the precise order of amino acids that give functionality to proteins. In all organisms, translational components are modified by the addition of one or more methyl groups. Ribosomal RNA methylation sites are clustered in functional regions of the ribosome, including the peptidyl transferase center and the tRNA binding sites (A/P/E), and have been shown to be important for ribosome assembly, structural stability, and translational accuracy (Piekna-Przybylska et al. 2008; Baudin-Baillieu et al. 2009). Methylation of tRNAs and elongation and release factors modulates translational accuracy and translation termination efficiency (Johansson and Byström 2005; Polevoda and Sherman 2007; Dzialo et al. 2014; Hori 2014). Ribosomal proteins are methylated in the three kingdoms of life, but little is known about the physiological roles of these modifications (Arnold and Reilly 1999; Lee et al. 2002; Odintsova et al.

Corresponding author: clarke@mbi.ucla.edu

Article published online ahead of print. Article and publication date are at http://www.rnajournal.org/cgi/doi/10.1261/rna.054569.115.
2003; Yu et al. 2005; Polevoda and Sherman 2007; Nesterchuk et al. 2011).

In Escherichia coli, methylation of ribosomal protein L3 by the PrmB methyltransferase has been shown to modulate ribosome biogenesis (Lhoest and Colson 1981; Lhoest et al. 1984). Escherichia coli L11 contains nine methyl groups in close proximity to the GTPase-associated center (GAC) and the binding site of translation factors, suggesting that they could play a role in translation (Dognin and WittmannLiebold 1980). Methylation of the bacterial L7/L12 stalk is regulated by the growth temperature, and it may play a regulatory role in homeostasis (Chang 1978). Methylation of human ribosomal proteins S3 and S10 by PRMT1 and PRMT5, respectively, has been implicated in roles in ribosomal biogenesis (Shin et al. 2009; Ren et al. 2010). In the yeast Saccharomyces cerevisiae, nine ribosomal proteins are methylated: four on the small ribosomal subunit (Rps2, Rps3, Rps25ab, Rps27ab) and five on the large subunit (Rpllab,

(c) 2016 Al-Hadid et al. This article is distributed exclusively by the RNA Society for the first 12 months after the full-issue publication date (see http://rnajournal.cshlp.org/site/misc/terms.xhtml). After 12 months, it is available under a Creative Commons License (Attribution-NonCommercial 4.0 International), as described at http://creativecommons.org/licenses/by$\mathrm{nc} / 4.0 /$. 
Rpl3, Rpl12ab, Rpl23ab, Rpl42ab) (Chern et al. 2002; Lee et al. 2002; Porras-Yakushi et al. 2005; Lipson et al. 2010; Webb et al. 2010a,b, 2011; Young et al. 2012). Unlike the clustering of methylated sites on rRNA nucleotides, the methylated residues on yeast ribosomal proteins are dispersed throughout the ribosome: seven are on the surface exposed to the cytoplasm, two are in the interface between the small (40S) and large (60S) ribosomal subunits, and three are embedded within the rRNA core (Clarke 2013).

Although the ribosomal protein methylation patterns differ in the three domains of life, methylation of two ribosomal proteins, Rpl3 and Rpl12ab, appears to be conserved. Yeast Rpl12ab and its bacterial ortholog L11 are methylated N-terminally and human Rpl12 is also predicted to be methylated at the N-terminus (Dognin and Wittmann-Liebold 1980; Webb et al. 2010b). In E. coli, L3 is methylated on a glutamine residue that aligns close to the methylated histidine site on Rpl3 in S. cerevisiae (Muranova et al. 1978; Webb et al. 2010a). Additionally, the mammalian Rpl3 associates with METTL18, the human putative methyltransferase homologous to the yeast Rpl3 methyltransferase Hpm1 (Cloutier et al. 2013). 3-Methylhistidine is a relatively rare post-translational modification that has only been observed in eukaryotes in Rpl3, actin, and myosin-Hpm1 is the first and as of yet only enzyme known to catalyze this modification (Webb et al. 2010a).

In a recent study, we showed that Hpm 1 directly methylates ribosome-bound Rpl3 but not free Rpl3, suggesting that it likely recognizes and methylates Rpl3 after its incorporation into pre-ribosomes (Al-Hadid et al. 2014). The proper processing of early pre-rRNA intermediates and assembly of the large ribosomal subunit was shown to be dependent on the presence of Hpm1. Loss of Hpm1 resulted in a large subunit biogenesis defect that could only be rescued with catalytically active Hpm 1 but not catalytically deficient mutants of Hpm1, suggesting that methylation of Rpl3 or other unknown substrates is required for proper $60 \mathrm{~S}$ biogenesis. Ribosomes assembled in Hpm1-deficient cells were also shown to have reduced sensitivities to ribosome-binding drugs and reduced translational accuracy. Whether these phenotypes were directly due to a lack of Rpl3 methylation or to other potential roles of Hpm 1 in methylating other substrates was not investigated.

In this study, we analyzed a mutant of Rpl3 with the methylated histidine replaced with alanine and determined the effect of Rpl3 methylation loss on ribosome biogenesis and translation. We show that unlike $h p m 1$ null cells, rpl3H243A mutants have normal levels of ribosomal subunits. In contrast, Hpm1-deficient cells and rpl3-H243A mutants both exhibit defects in early pre-rRNA processing and have higher rates of translational errors in stop codon readthrough, amino acid misincorporation, and programmed - 1 ribosomal frameshifting (-1 PRF). Amino acid analysis of the ${ }^{3} \mathrm{H}$-methyl-labeled proteome and in vitro methylation assays indicate that Hpm1 has additional substrate(s) in different subcellular compartments. Altogether, these results suggest that methylation of Rpl3 at histidine 243 modulates translation elongation fidelity and indicates that $\mathrm{Hpm} 1$ has independent functions in ribosome biogenesis and translation.

\section{RESULTS AND DISCUSSION}

\section{Histidine 243 of ribosomal protein Rpl3 is the target of the Hpm1 methyltransferase}

In previous work, we pinpointed the Hpml target site on Rpl3 to an 18-residue region (residues 234-251) containing multiple lysine and arginine residues (Webb et al. 2010a). To determine the methylated amino acid, wild-type yeast cells were labeled with $\left[\right.$ methyl- $\left.{ }^{3} \mathrm{H}\right]$ AdoMet, ribosomes harvested, Rpl3 was isolated by reversed-phase HPLC, and the protein acid hydrolyzed. The amino acids were resolved by high-resolution cation-exchange and thin layer chromatography. A radiolabeled species co-eluted with a 3-methylhistidine standard but not with methylated lysine or arginine standards, suggesting that the modification is on a histidine residue. To definitively show that $\mathrm{H} 243$ is the methylated residue on Rpl3, we created an rpl3 mutant strain by replacing the endogenous RPL3 gene with an rpl3 point mutant containing a histidine to alanine mutation at residue 243 (rpl3$H 243 A)$. WT, hpm1s, and rpl3-H243A cells were labeled with $\left[\right.$ methyl- $\left.{ }^{3} \mathrm{H}\right]$ AdoMet and the small and large ribosomal subunits were isolated by sucrose density centrifugation. Radiolabeled proteins were resolved by SDS-PAGE and methylated products detected by fluorography (Fig. 1A). Small ribosomal subunit proteins had similar methylation patterns in all three strains. However, large ribosomal subunit proteins showed a complete loss of a radiolabeled protein corresponding to the size of Rpl3 $(43.8 \mathrm{kDa})$ in both hpm1 1 and rpl3-H243A cells but not in wild-type cells, confirming that $\mathrm{H} 243$ of Rpl3 is the methylation target of Hpm1. $\mathrm{H} 243$ lies at the core of the large subunit embedded in the $25 \mathrm{~S}$ rRNA in close proximity to the peptidyl transferase center and the aminoacyl-tRNA accommodation corridor (Fig. 1B).

\section{Methylation of ribosomal protein Rpl3 is dispensable for 605 subunit synthesis}

We previously showed that loss of $\mathrm{Hpm} 1$ results in pre-rRNA processing defects in the early stages of ribosome biogenesis leading to a deficiency of 60S large ribosomal subunits (Al-Hadid et al. 2014). Proper 60S biogenesis was shown to depend not only on the Hpml protein but also its catalytic activity, suggesting that methylation of its primary target, $\mathrm{Rpl3}$, and/or of other substrates, is involved in promoting proper ribosome biogenesis. To test whether the phenotypes we observed were strictly a consequence of the loss of Rpl3 methylation rather than due to the lack of methylation of other potential substrates of Hpm1, we examined the steady-state levels of pre-rRNA precursors and ribosome particles in the Rpl3 methylation-deficient strains. Total cellular 
A

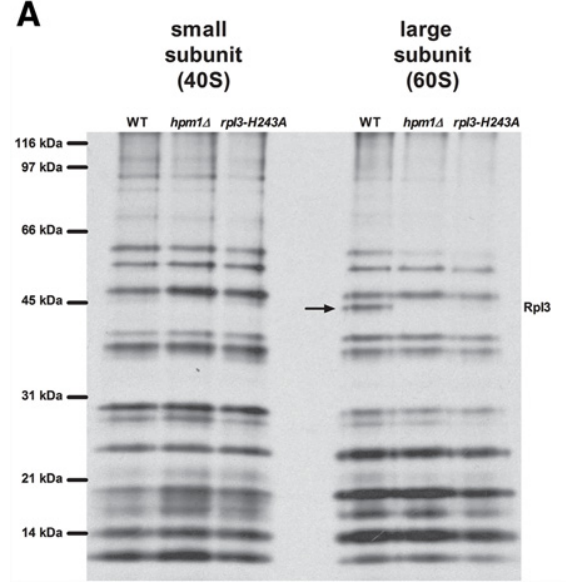

B

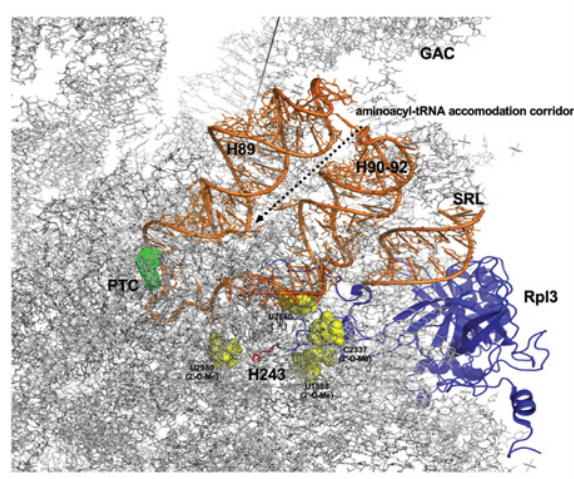

FIGURE 1. Rpl3 is methylated at histidine 243 , which lies in close proximity to functional centers of the ribosome. (A) Seven $\mathrm{OD}_{600 \mathrm{~nm}}$ units of WT, $h p m 1 \Delta$, and $r p l 3-\mathrm{H} 243 \mathrm{~A}$ cells were radiolabeled as described in Materials and Methods. Small (40S) and large (60S) ribosomal subunits were separated by sucrose density centrifugation and radiolabeled proteins resolved and detected by SDSPAGE/fluorography using a 12\% Bis-Tris gel as described by Al-Hadid et al. (2014). Fluorography was done for $10 \mathrm{wk}$ at $-80^{\circ} \mathrm{C}$. The fluorograph shown is the same as that in Fig. 1C of Al-Hadid et al. (2014) except it is uncropped and includes ribosomal subunits from the rpl3-H243A strain. (B) Crystal structure of the yeast ribosome (Ben-Shem et al. 2011) showing Rpl3 (blue) with its H243 residue (red) embedded in 25S rRNA (gray). Helixes 89 and 90-92 (orange) form the entry site for aminoacyl-tRNAs. The GTPase-associated center (GAC) and sarcin-ricin loop (SRL; orange) are the binding sites of translation factors. Residues of the peptidyl transferase center (PTC) are shown as green spheres. Yellow spheres show modified nucleotides (2'-OH methylation, and pseudouridine $\psi$ ) of $25 \mathrm{~S}$ rRNA in close proximity to $\mathrm{H} 243$.

RNAs were collected from WT, $h p m 1 \Delta$, and $r p l 3-H 243 A$ cells and analyzed by Northern blotting (Fig. 2A). A DNA probe that binds upstream of the A0 cleavage site was used to detect the $35 \mathrm{~S}$ and $23 \mathrm{~S}$ rRNA precursors that are known to accumulate in mutants defective in pre-rRNA processing (Oeffinger et al. 2009; Woolford and Baserga 2013). Both Hpm1-deficient cells and rpl3-H243A mutants showed similar accumulation of $35 \mathrm{~S}$ and $23 \mathrm{~S}$ precursors compared to wild type, whereas no significant differences were detected in the levels of 27SA2 precursors (Fig. 2A). Ribosome assembly proceeds cotranscriptionally with the small subunit factors assembling on the $18 \mathrm{~S}$ sequence, before transcription of the $5.8 \mathrm{~S}$ and $25 \mathrm{~S}$ sequences (Venema and Tollervey 1999). Cleavage at site A2, which separates the $18 \mathrm{~S}$ and $5.8 \mathrm{~S}$ rRNAs, occurs after $18 \mathrm{~S}$ association with small subunit factors and before the association of large subunit factors with the $5.8 \mathrm{~S}$ and $25 \mathrm{~S}$ (Grandi et al. 2002). Counterintuitively, defects in assembly and processing of the pre-60S large subunit result in defects in the earliest $35 \mathrm{~S}$ processing steps at $\mathrm{A} 0, \mathrm{~A} 1$, and $\mathrm{A} 2$, most likely through indirect secondary effects (van Beekvelt et al. 2001; Venema and Tollervey 1999 and references therein). Therefore, it is likely that the effect of $h p m 1 \Delta$ and rpl3$H 243 \mathrm{~A}$ on $35 \mathrm{~S}$ pre-rRNA processing is indirect, consistent with previous studies on other large subunit biogenesis factors (Venema and Tollervey 1999). It is possible that kinetic defects in large subunit biogenesis may sequester factors that are shared between pre-60S and pre-90S maturation, such as Nop1p, Sik1p, and Rrp5p (Venema and Tollervey 1996;

Gautier et al. 1997). However, precisely how specific 605 maturation defects communicate back to early processing steps is unknown. Indeed, many large subunit ribosomal proteins, including $\mathrm{Rpl3}$, associate with the $25 \mathrm{~S}$ cotranscriptionally and are known to directly impact processing (Rosado et al. 2007; Henras et al. 2015). Despite an accumulation of the $35 \mathrm{~S}$ precursor, we did not detect any significant difference in the final levels of mature $25 \mathrm{~S}$ and $18 \mathrm{~S}$ rRNAs in hpm1s and rpl3-H243A (Fig. 2A). This indicates that these processing defects are not rate limiting for the maintenance of mature rRNA levels, at least during steady-state growth in rich medium.

To determine whether the increased levels of $35 \mathrm{~S}$ and $23 \mathrm{~S}$ prerRNA in rpl3-H243A result in a $60 \mathrm{~S}$ biogenesis defect, the steady-state levels of ribosomal subunits and ribosome complexes were measured by polysome profile analysis. Small (40S) and large (60S) ribosomal subunits were resolved from intact ribosomes (80S) and polysomes by centrifugation of the lysates from wild-type, $h p m 1 \Delta$, and $r p l 3-H 243 A$ strains through a sucrose gradient and the levels of the ribosomal particles were measured. As previously shown (Al-Hadid et al. 2014), hpm1 null cells exhibited a deficiency of $60 \mathrm{~S}$ subunits and a surplus of $40 \mathrm{~S}$ subunits (Fig. 2B). This large subunit defect is highlighted by a greater than fourfold decrease in the free $60 \mathrm{~S} /$ free $40 \mathrm{~S}$ subunit ratio (Fig. 2C). Surprisingly, rpl3-H243A showed levels of the ribosomal particles similar to wild type, with no discernable difference in their respective free $60 \mathrm{~S} /$ free $40 \mathrm{~S}$ subunit ratios (Fig. 2B,C). This indicates that despite a pre-rRNA processing defect in rpl3-H243A, steady-state levels of free ribosomal subunits are unchanged. These results suggest that methylation of Rpl3 is required for normal pre-rRNA processing but not for accumulating normal levels of 605 subunits. This result was unexpected because we previously showed that Rpl3 is the primary target of $\mathrm{Hpm} 1$ and that the $\mathrm{Hpm} 1$ protein and its catalytic activity are required for normal ribosome biogenesis (Al-Hadid et al. 2014). It is therefore likely that Hpml has additional substrates whose methylation plays a role in translation elongation and/or ribosome biogenesis.

Interestingly, $h p m 1 \Delta$ and $r p l 3-H 243 A$ cells had marginally higher polysome/80S ratios, compared to wild-type cells, indicating that a higher proportion of ribosomes may be associated with polysomes in these mutants (Fig. 2C). Although a larger fraction of polysome-associated ribosomes is typically indicative of more active translation and 
A

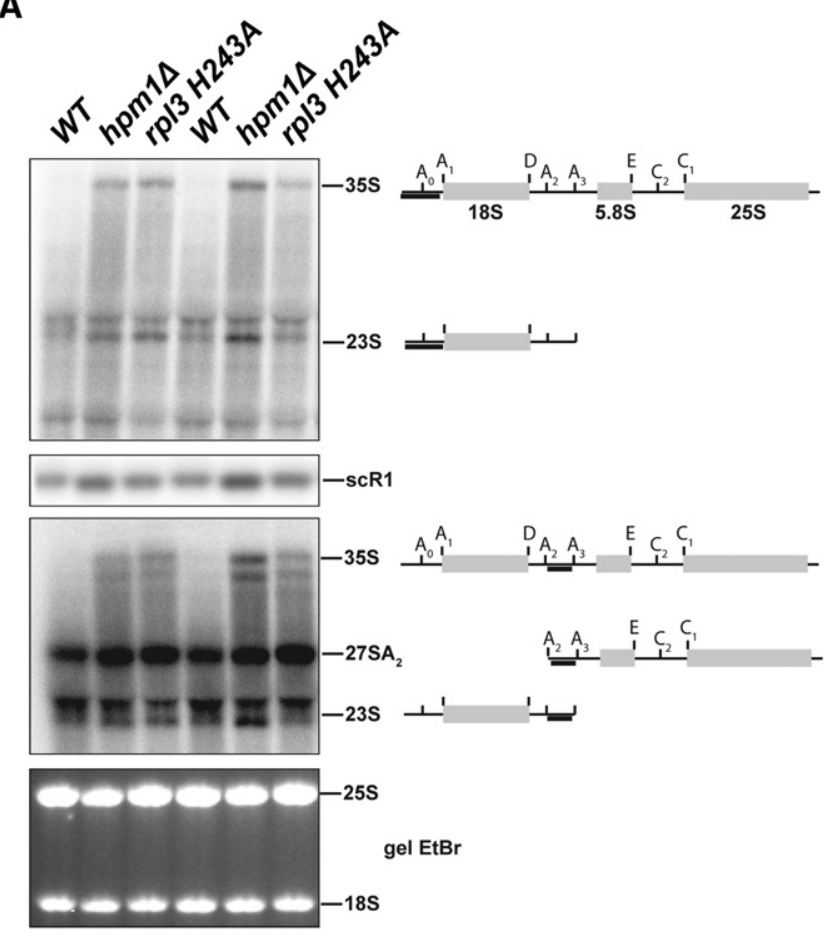

C

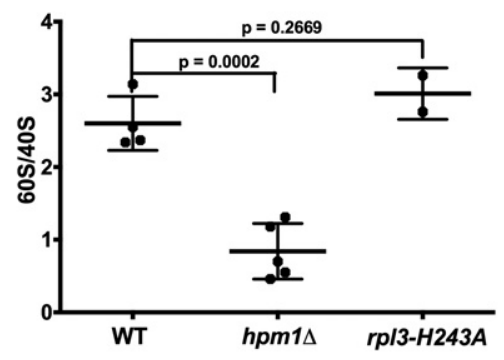

B
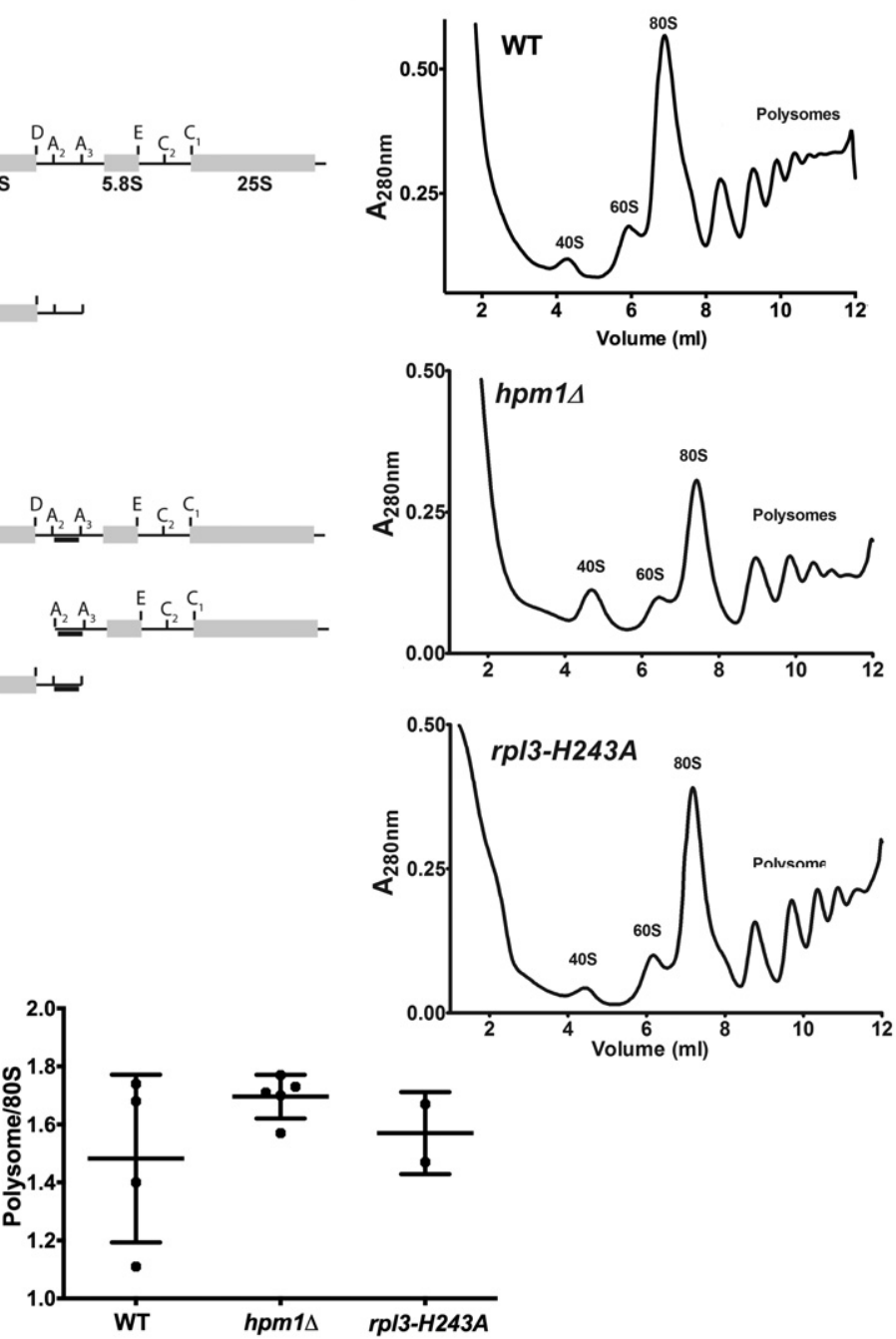

FIGURE 2. Loss of Rpl3 methylation results in early pre-rRNA processing defects but has no effect on $60 \mathrm{~S}$ subunit levels. (A) $35 \mathrm{~S}$ and $23 \mathrm{~S}$ rRNA levels were assessed by Northern blot analysis as previously described (Al-Hadid et al. 2014) in WT, $h p m 1 \Delta$, and $r p l 3-H 243 A$ cells with a probe $5^{\prime}$-CGCTGCTCACCAATGG-3' targeted to the region upstream of the A0 cleavage site in the $5^{\prime}$ external transcribed spacer of the rRNA precursor (upper blot). A probe targeting the internal transcribed spacer 1 (ITS1) region in between the A2 and A3 cleavage sites (O207: 5'-TGTTACCTCTGGGCCC-3') was used to detect the 27SA2 precursor (lower blot). The 25S and 18S rRNAs are shown as visualized by EtBr fluorescence of the gel prior to transfer. The second series of strains in the blot represent biological replicates, with the experiment performed for a total of four biological replicates. scR1 (5'-ATCCCGGCCGCCTCCATCAC-3') is a loading control. (B) WT, $h p m 1 \Delta$, and $r p l 3-H 243 A$ cells were treated with cycloheximide and lysates resolved by sucrose density centrifugation for polysome profile analysis as previously described (Al-Hadid et al. 2014). A total of four independent profiles were obtained for wild-type cells, five profiles for $h p m 1 \Delta$ cells, and two profiles for $r p l 3-H 243 A$ cells. Two of the profiles for wild-type and $h p m 1 \Delta$ cells were previously reported (Al-Hadid et al. 2014). A representative profile for each strain is shown here. (C) The levels of ribosomal subunits $(40 \mathrm{~S}, 60 \mathrm{~S})$ and intact ribosomes $(80 \mathrm{~S})$ were quantified by directly weighing cutouts of half peak areas (assuming a symmetric distribution of ribosome particles) from printer paper using an analytical balance. The levels of polysomes were quantified by weighing the peaks after the $80 \mathrm{~S}$. Error bars represent standard deviation of two or more independent profiles. Unpaired two-tailed $t$-test $P$-values are shown for the differences in the $60 \mathrm{~S} / 40 \mathrm{~S}$ free subunit ratio.

cellular proliferation, we found that growth rates on YPD agar plates or liquid cultures (data not shown) were similar in all three strains. Indeed, increased polysome levels have been reported in cells with attenuated translation elongation, resulting in increased mRNA transit time of ribosomes and stabilization of translationally repressed polysomes (Sivan et al. 2007).

\section{Hpm1 has methylation targets other than Rpl3}

We previously showed that in the absence of Hpm1, methylation was lost not only in Rpl3 but also in other proteins in nuclei-enriched fractions, indicating that there could be additional substrates for Hpm1 (Al-Hadid et al. 2014). Our repeated attempts to create an endogenous TAP-tagged 
Hpm1 strain or express GFP-tagged Hpm1 ectopically for pull-down studies were unsuccessful. In the latter case, the recombinant protein underwent rapid degradation. As an alternative approach to determine whether there are additional Hpm1 substrates, the cellular levels of 3-methylhistidine were measured in wild-type and rpl3-H243A cells. We previously showed that Hpml depletion results in complete loss of 3-methylhistidine. If Rpl3 is the only substrate of Hpm1, there should be a complete loss of 3-methylhistidine in rpl3-H243A cells, because the methylation site is mutated. Wild-type and $r p l 3-H 243 A$ cells were radiolabeled with [methyl- $\left.{ }^{3} \mathrm{H}\right]$ AdoMet and cellular proteins were precipitated with trichloroacetic acid, acid hydrolyzed, and analyzed by high-resolution cation-exchange chromatography. Fractions containing radioactive 3 -methylhistidine were identified by ninhydrin labeling of a nonradioactive 3 -methylhistidine standard and counted for radioactivity. The rpl3-H243A lysate showed a significant decrease in the amount of 3-methylhistidine, relative to wild type, although a residual amount was still present (Fig. 3A). This residual radioactivity therefore represents 3-methylhistidine present on a protein or proteins other than Rpl3. Quantification of the levels of 3-methylhistidine in wild-type and rpl3-H243A lysates revealed that $\sim 65 \%$ of the modification resides on $\mathrm{Rpl} 3$ and the remaining amount is present on other substrates. This is consistent with our previous finding that the bulk of 3methylhistidine is on ribosome-associated Rpl3 (Al-Hadid et al. 2014).

To determine whether there are additional substrates of Hpm1, in vitro and in vivo methylation assays were performed on subcellular fractions from wild-type, $h p m 1 \Delta$, and rpl3-H243A cells. Cells were radiolabeled with $\left[\right.$ methyl- $\left.{ }^{3} \mathrm{H}\right]$ AdoMet and cytosolic and nuclear proteins isolated and resolved by SDS-PAGE. Methylated polypeptides were detected by fluorography. Loss of $\mathrm{Hpm} 1$ resulted in the reduction or complete loss of seven radiolabeled bands from the cytosol (Fig. 3B, arrows, stars). These bands were not reduced in cytosolic proteins from rpl3-H243A cells, indicating that they are not Rpl3-derived polypeptides (Fig. $3 \mathrm{~B}$ ). This suggests that the methylation of multiple proteins may depend on the presence of Hpm1.

Analysis of nuclear proteins revealed reduction of a radiolabeled band corresponding to the size of Rpl3 in $h p m 1 \Delta$ and rpl3-H243A cells (Fig. 3C, arrow). Several additional lower molecular weight bands were also absent or diminished in rpl3-H243A, suggesting that these are Rpl3-derived polypeptides (Fig. 3C). To distinguish proteins that are directly methylated by Hpml from those whose methylation depends on Hpml but are not necessarily substrates, we performed an in vitro methylation assay using recombinant His-tagged Hpm1. Nuclear and post-mitochondrial extracts (supernatant following 20,000g spin) from wild-type and Hpm1-deficient cells were incubated with His-Hpml in the presence of [methyl- $\left.{ }^{3} \mathrm{H}\right]$ AdoMet and analyzed by SDS-PAGE fluorography. Protein substrates in $h p m 1 \Delta$ would be hypomethylated and competent for methylation with $\mathrm{Hpm} 1$ and $\left[\right.$ methyl $\left.-{ }^{3} \mathrm{H}\right]$ AdoMet, whereas wild-type proteins may not be because they may already be fully or partially modified. In vitro methylation of nuclei-enriched fractions from $h p m 1 \Delta$ cells but not wild-type cells resulted in the radiolabeling of an Rpl3-sized protein (Fig. 3E, star). We previously showed that Rpl3 from cytoplasmic ribosomes could be methylated in vitro with recombinant Hpml (Al-Hadid et al. 2014). Together, this suggests that $\mathrm{Rpl} 3$ bound to pre-ribosomes in the nucleus or mature ribosomes in the cytoplasm can be recognized and methylated by Hpm1. Interestingly, incubating His-Hpm1 with post-mitochondrial extracts from $h p m 1 \Delta$ but not wild type resulted in the radiolabeling of two proteins (Fig. 3D, stars). The electrophoretic mobility of these proteins is similar to two of the in vivo methylated cytosolic proteins in the wild-type strain (Fig. 3B, stars). Although the identity of these substrates is unknown, these data suggest that Hpml has multiple substrates; one corresponding to $\mathrm{Rpl} 3$ that can be methylated in nuclear extracts and two that can be methylated in post-mitochondrial extracts. It is unclear if one of the radiolabeled bands in the post-mitochondrial extract is Rpl3. However, because the intensities of the two bands are similar and have similar mobilities to two non-Rpl3 proteins in the in vivo labeled cytosolic proteins (Fig. 3B, stars), these data suggest that the two radiolabeled bands in the post-mitochondrial extract do not correspond to Rpl3. It is also possible that methylation of these unknown proteins may be important for ribosome assembly.

Despite cytoplasmic ribosomes containing the bulk of 3-methylhistidine, our previous findings that Hpm1-deficient cells have defects in early pre-rRNA processing, which takes place in the nucleus, and the lack of detectable levels of 3-methylhistidine in the cytosol suggests that the modification of Rpl3 by Hpm 1 takes place within the nucleus. It is possible that the modification of the two other proteins takes place in the cytosol but that their modification is undetectable in cytosolic fractions because of the rapid shuttling of these proteins into the nucleus. Further work is needed to identify these proteins and their spatial and temporal methylation.

\section{Methylation of ribosomal protein Rpl3 is required for translational accuracy}

The dispensability of Rpl3 methylation in 60S subunit biogenesis prompted us to test whether methylation of Rpl3 plays a role in translational fidelity. Using a dual-luciferase reporter assay, we previously showed that Hpm1-deficient cells have decreased fidelity during translation elongation (Al-Hadid et al. 2014). This assay utilizes a Renilla luciferase reporter gene fused C-terminally to a firefly luciferase gene (Keeling et al. 2004; Salas-Marco and Bedwell 2005). The amount of firefly luciferase luminescence correlates with increased translational errors, while Renilla luminescence is used to normalize for differences in gene expression and translational initiation, as both genes are expressed as a single polypeptide. 
A

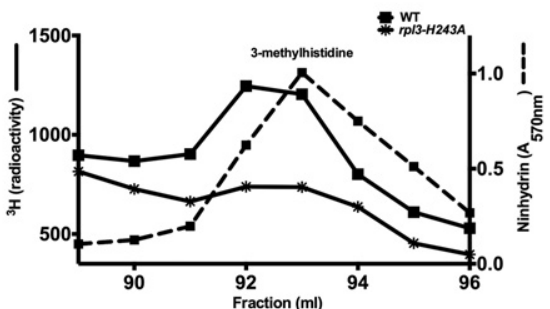

C

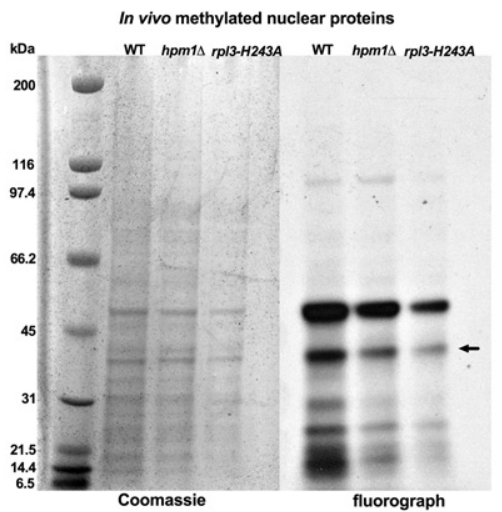

B

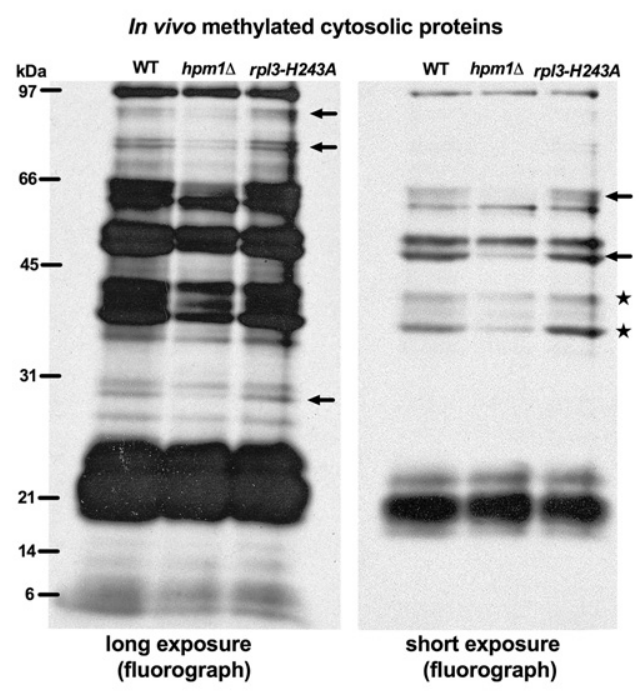

E

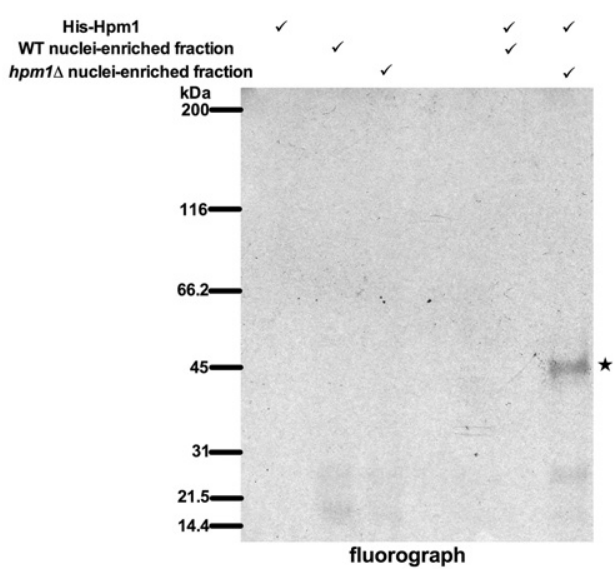

FIGURE 3. Hpml has multiple substrates. (A) WT and $r p l 3-H 243 A$ cells were radiolabeled with $\left[\right.$ methyl $\left.{ }^{3} \mathrm{H}\right]$ AdoMet as described in Materials and Methods. Cell pellets were resuspended in $200-400 \mu \mathrm{L}$ of buffer (50 mM Tris-Cl, $50 \mathrm{mM} \mathrm{NaCl}, 1 \mathrm{mM} \mathrm{DTT}$, pH 7.4), a quarter volume of glass beads was added, and the cells were vortexed for $30 \mathrm{sec}$ followed by $30 \mathrm{sec}$ cooling on ice, for 12 cycles. The crude lysate was centrifuged at $1000 \mathrm{~g}$ for $5 \mathrm{~min}$ and the supernatant transferred to prechilled microcentrifuge tubes (whole lysate). Radiolabeled whole lysates were precipitated with trichloroacetic acid, acid hydrolyzed, and amino acids resolved by cation-exchange chromatography (Al-Hadid et al. 2014). Fractions containing ${ }^{3} \mathrm{H}-3$-methylhistidine were identified using ninhydrin monitoring of an unlabeled 3-methylhistidine standard (dashed line), and counting for radioactivity (solid lines; squares for WT, asterisks for $r p l 3-H 243 A$ ). Radiolabeled 3-methylhistidine elutes 1 min earlier than the unlabeled standard due to a known isotope effect (Gottschling and Freese 1962). (B) One hundred microliters of fraction 1 (cytosol) of the sucrose gradient from WT, $h p m 1 \Delta$, and rpl3-H243A cells were resolved by SDS-PAGE using a 12\% Bis-Tris gel (Al-Hadid et al. 2014). The short exposure fluorograph was incubated for $13 \mathrm{~d}$ with the radiolabeled gel and the film with the long exposure was incubated for $10 \mathrm{wk}$, both at $-80^{\circ} \mathrm{C}$. Arrows and stars show radiolabeled bands that are lost in $h p m 1 \Delta$ but not in $r p l 3-H 243 A$, indicative of protein substrates other than Rpl3 whose methylation depends on HPM1. Stars indicate radiolabeled bands with similar mobilities as those shown in panel $D$. (C) $14 \mathrm{OD}_{600 \mathrm{~nm}}$ units of WT, $h p m 1 \Delta$, and $r p l 3-H 243 A$ cells were radiolabeled with $\left[\right.$ methyl- $\left.{ }^{3} \mathrm{H}\right]$ AdoMet and nuclei-enriched fractions $\left(\mathrm{P}^{13,000}\right)$ isolated as described in Materials and Methods. Samples were treated with 1 unit of benzonase with $2 \mathrm{mM}$ magnesium chloride for $30 \mathrm{~min}$ at room temperature. Fifteen micrograms of nuclear-enriched extract from WT, $h p m 1 \Delta$, and rpl3-H243A cells were then resolved and analyzed by SDS-PAGE/fluorograph as described above. Film was incubated for $29 \mathrm{~d}$ with gel at $-80^{\circ} \mathrm{C}$. Arrow shows a radiolabeled protein corresponding to the size of Rpl3 that is lost in $h p m 1 \Delta$ and $r p l 3-H 243 A$. (D) Forty OD $600 \mathrm{~nm}$ units of WT and $h p m 1 \Delta$ cells were harvested, spheroplasts prepared, and post-mitochondrial cell extracts $\left(S^{20,000}\right)$ isolated as described in Materials and Methods. Twenty micrograms of recombinant histidine-tagged $\mathrm{Hpm} 1$ (His-Hpml) were incubated alone or with $50 \mu \mathrm{g}$ of post-mitochondrial cell extracts from WT and hpm $1 \Delta$ cells in the presence of $\left[\right.$ methyl- $\left.^{3} \mathrm{H}\right]$ AdoMet $(1 \mu \mathrm{M})$ and $100 \mathrm{mM}$ sodium chloride, $100 \mathrm{mM}$ sodium phosphate, pH 7.5 (methylation buffer) for $6 \mathrm{~h}$ at $30^{\circ} \mathrm{C}$. Wild-type and $h p m 1 \Delta$ post-mitochondrial cell extracts without enzyme were also included for negative controls. Samples were then treated with 1 unit of benzonase (Novagen; 70746-4) for $30 \mathrm{~min}$ at room temperature to degrade nucleic acids and the volume reduced by vacuum centrifugation. Samples were then loaded onto 4\%-12\% SDS-PAGE gel (ExpressPlus; GenScript) using Tris-MOPS (SDS) running buffer and proteins resolved by applying $150 \mathrm{~V}$ until the bromophenol blue dye reached the bottom of the gel. Fluorography was performed and the film was incubated with gel for $8 \mathrm{~d}$ at $-80^{\circ} \mathrm{C}$. Stars indicate $\mathrm{Hpm} 1$-methylated protein substrates. Vertical black lines show where irrelevant lanes were removed from the gel. (E) Nuclei-enriched fractions were isolated from wild-type and hpm $1 \Delta$ cells as described in Al-Hadid et al. (2014). Twelve micrograms of recombinant His-Hpml were incubated alone or with $23 \mu \mathrm{g}$ of nuclei-enriched fractions from wild type and $h p m 1 \Delta$ in the presence of [methyl- $\left.{ }^{3} \mathrm{H}\right]$ AdoMet $(1 \mu \mathrm{M})$ and methylation buffer for $5 \mathrm{~h}$ at $30^{\circ} \mathrm{C}$. Samples were treated with 1 unit of benzonase for $30 \mathrm{~min}$ at $30^{\circ} \mathrm{C}$. Proteins were resolved in a $4 \%-12 \%$ SDS-PAGE gel (Run Blue) and radiolabeled proteins detected by fluorography as described above. Film was exposed for $11 \mathrm{~d}$ at $-80^{\circ} \mathrm{C}$. The star indicates an Hpml-methylated protein product corresponding to the size of Rpl3 ( $44 \mathrm{kDa})$. 
To measure stop codon readthrough, which can arise due to defects in translation elongation or termination, we used reporter plasmids containing the UAA and UAG stop codons in between the Renilla and firefly genes (Keeling et al. 2004; Salas-Marco and Bedwell 2005). Increased readthrough of these stop codons would result in increased firefly luminescence. Both Hpm1-deficient cells, as previously shown (AlHadid et al. 2014), and Rpl3 methylation-deficient cells (rpl3-H243A) exhibited a minor increase in readthrough of the UAA and UAG stop codons, relative to wild type (Fig. $4 \mathrm{~A})$. To determine whether this stop codon readthrough phenotype in rpl3-H243A is due to a defect in translation termination or elongation, a reporter plasmid was used that directly measures elongation errors by the rate of amino acid misincorporation. This plasmid contains a mutation at K529 of the firefly gene to a near-cognate asparagine residue, which renders the firefly enzyme catalytically inactive (SalasMarco and Bedwell 2005). Replacement of this asparagine back to the wild-type lysine by misincorporation of a nearcognate lysyl-tRNA ${ }^{\text {Lys }}$ reestablishes the catalytic activity of firefly luciferase and results in an increase in its luminescence. Thus, ribosomes with a reduced ability to discriminate between cognate and near-cognate aminoacyl-tRNAs during translation elongation yield higher levels of firefly luciferase luminescence. Both $h p m 1 \Delta$ and $r p l 3-H 243 A$ cells showed slightly increased levels of amino acid misincorporation, relative to wild type, indicating a loss of fidelity in both mutants (Fig. 4B).

To further characterize translation elongation in $h p m 1 \Delta$ and Rpl3 methylation-deficient cells, translation recoding efficiency was measured using a reporter containing a viral L-A directed programmed -1 frameshift (-1 PRF) signal between the Renilla and firefly genes (Harger and Dinman 2003). The amount of firefly luciferase luminescence depends on the frequency of -1 frameshift events. The frequency of -1 frameshift events is essential to the propagation of the L-A virus as it relies on a specific stoichiometry of structural (Gag) to enzymatic (pol) proteins encoded in its $4.6 \mathrm{~kb}$ dsRNA genome. Defects in elongation have been shown to enhance -1 PRF efficiencies (Peltz et al. 1999; Meskauskas et al. 2003, 2005). Hpm1-deficient and Rpl3 methylation-deficient cells showed higher -1 PRF efficiencies, relative to wild type (Fig. 4C). These results suggest that the defect in translation elongation fidelity that we previously reported for Hpm1deficient cells is a direct consequence of a lack of Rpl3 methylation. We should point out, however, that Rpl3 in the rpl3-H243A strain contains an alanine residue at position 243 and some of the translational defects may be due to the loss of the histidine side chain as well as loss of the methylation. This may explain why the rpl3-H243A mutant exhibited a more pronounced increase in stop codon readthrough, amino acid misincorporation, and recoding efficiency than Hpm1-deficient cells.

The stop codon readthrough phenotype is likely a consequence of decreased accuracy during translation elongation,

\section{A}

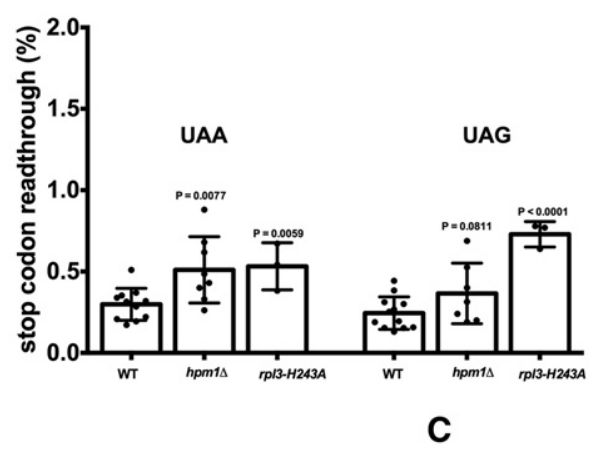

B
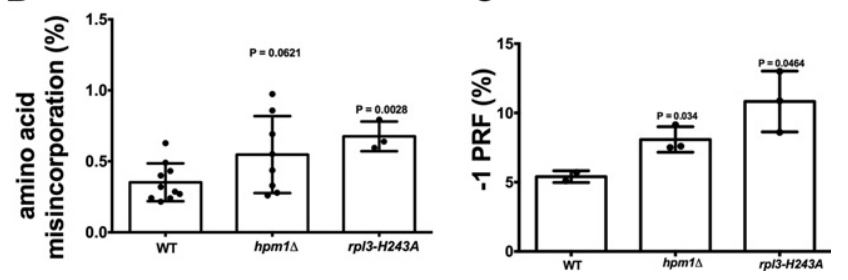

FIGURE 4. Cells deficient in Rpl3 methylation have reduced translational fidelity. Dual-luciferase assays were performed to quantify stop codon readthrough, amino acid misincorporation, and programmed -1 ribosomal frameshifting (-1 PRF), as described in Al-Hadid et al. (2014), for WT, $h p m 1 \Delta$, and $r p l 3-H 243 A$ cells. (A) The percent readthrough of the UAA and UAG stop codons was calculated by taking the firefly/Renilla luminescence ratio of cells containing the stop codon-containing vector divided by the same ratio of the respective control. For UAA readthrough, wild type was assayed 11 independent times, $h p m 1 \Delta$ eight times, and $r p l 3-H 243 A$ was assayed three independent times. Three of these replicates for wild-type and $h p m 1 \Delta$ cells were previously reported (Al-Hadid et al. 2014). For UAG readthrough, wild type was assayed for a total of 12 biological replicates, $h p m 1 \Delta$ was assayed seven times, and $r p l 3-H 243 A$ three times. Three of these replicates for wild-type and $h p m 1 \Delta$ cells were previously reported (AlHadid et al. 2014). (B) The percent amino acid misincorporation was calculated as in $A$. Wild type was assayed 10 independent times, $h p m 1 \Delta$ was assayed eight times, and $r p l 3-H 243 A$ was assayed three independent times. Three of these replicates for wild-type and $h p m 1 \Delta$ cells were previously reported (Al-Hadid et al. 2014). (C) The percent -1 PRF was calculated by taking the firefly/Renilla luminescence ratio of cells containing pJD376 (L-A virus gag-pol frameshift signal) divided by the same ratio of the no frameshift control (pJD375). Error bars represent the standard deviation. Unpaired two-tailed $t$-test $P$-values are shown for the differences in translational fidelity in wild-type and mutant strains.

in which the ability of the ribosome to discriminate between cognate and near-cognate or non-cognate aminoacyl-tRNAs is diminished, as has been reported (Salas-Marco and Bedwell 2005). Rpl3 methylation-deficient cells also had enhanced recoding efficiencies of -1 PRF signals, a phenotype that can arise due to defects in translation elongation (Meskauskas et al. 2003). Our data demonstrate a possible increased proportion of polysomes in rpl3-H243A and $h p m 1 \Delta$, relative to wild type. Prior work has shown that polysomes can accumulate in cells with repressed translation elongation due to an increased transit time of the defective ribosomes on mRNA (Sivan et al. 2007). We suggest that an increased polysome ratio may be a direct consequence of the translation elongation defects in $r p l 3-H 243 A$ and $h p m 1 \Delta$. Altogether, 
these results suggest that $\mathrm{Rpl} 3$ methylation-deficient cells have defects in translation elongation.

\section{Conclusions}

We have used the rpl3-H243A mutant as a tool to assay the loss of function of a methylated histidine in cis on Rpl3, to complement our findings with the deletion of its cognate methyltransferase Hpm1. We cannot formally exclude the possibility that the histidine to alanine mutation affects Rpl3 function in a manner distinct from the nonmethylated histidine that exists in cells lacking Hpm1. However, we note that both Hpm1-deficient cells, which have a nonmethylated histidine at position 243 of Rpl3, and the rpl3-H243A mutant have similar pre-rRNA processing and translation elongation defects, while in polysome analysis, the rpl3-H243A mutant behaves more like the wild-type strain. Taken together, we conclude that it is the loss of methylation at histidine 243 of $\mathrm{Rpl} 3$ that is responsible for the translation elongation defects in both of these strains, rather than the change of side chain per se. Furthermore, we previously showed that Rpl3 is the only ribosomal substrate of $\mathrm{Hpml}$ (Al-Hadid et al. 2014); therefore it is likely that the translation elongation defects in hpm1 null cells is caused by loss of $\mathrm{Rpl} 3$ methylation. However, we cannot rule out that loss of methylation in the other nonribosomal substrates of Hpm1, which could be translation factors, causes or enhances the translational defects of hpm1 null cells.

$\mathrm{H} 243$ lies in a functionally important region of Rpl3 called the "basic thumb" that is proposed to synchronize the steps of translation elongation (Meskauskas and Dinman 2010). This basic thumb is a positively charged region, perpendicular to the tryptophan finger, proposed to function as a molecular clamp, linking functionally relevant rRNA domains through noncovalent interactions. The linkage of these rRNA domains, via the basic thumb, may enable the allosteric communication between the events taking place in the decoding center, the aminoacyl-tRNA accommodation corridor, the peptidyl transferase center, and the GTPase-associated center, which is paramount for the ability of the ribosome to select the correct aminoacyl-tRNA from the large pool of aa-tRNAs, and allow the precise amino acid to be added to the growing polypeptide chain. The structural integrity of the basic thumb is therefore crucial for this allosteric communication and thus the accuracy of translation. Loss of histidine methylation in Rpl3 is likely reducing the functionality of the basic thumb. We propose that methylation of $\mathrm{Rpl} 3$ at $\mathrm{H} 243$ promotes proper function of the basic thumb and the coordination of the decoding, peptidyl transfer, and translocation steps of translation elongation. Future structural, biochemical, and biophysical characterization of ribosomes from $\mathrm{Hpm} 1$-deficient cells and rpl3-H243A will be needed to fully understand how $\mathrm{H} 243$ methylation promotes fidelity during translation elongation.

\section{MATERIALS AND METHODS}

\section{Strains, growth media, recombinant protein expression, and purification}

All strains used in this study are of the BY4742 background (MATa, his $3 \Delta 1$; leu $2 \Delta 0$; lys $2 \Delta 0$; ura3 $\Delta 0$ ) obtained from the Open Biosystems yeast knockout collection (Thermo Scientific). hpm1 $\mathrm{p}$ contains a kanamycin resistance marker in place of the HPM1 open reading frame. Yeast strains were grown in $1 \%$ yeast extract, $2 \%$ peptone, and 2\% dextrose (YPD, Difco) or minimal synthetic defined media lacking uracil (SD -ura) containing $0.17 \%$ yeast nitrogen base without ammonium sulfate and amino acids (BD Biosciences), 0.077\% complete supplement mixture without uracil (MP Biomedicals; 114511212), 0.5\% ammonium sulfate (Acros; 423400010), and $2 \%$ dextrose (Fisher; D16-1).

The rpl3-H243A point mutant was constructed at the endogenous chromosomal locus in WT BY4742. First, the RPL3 open reading frame and its endogenous promoter and terminator were amplified from genomic DNA using primers 5'-GACTGACTACTAGTGGG ATTAACGCATTTCAGACAGC- $3^{\prime}$ and $5^{\prime}$-GACTGACTATCGAT AACCAGGGTGATGTAACAGAGG- $3^{\prime}$ and cloned into the SpeI and ClaI sites of the pUG35 plasmid (URA+). Restriction enzyme recognition sites are underlined. Next, the H243A mutation was introduced into the plasmid using the Stratagene QuikChange Lightning Site-Directed Mutagenesis kit (Agilent Technologies) using primers 5'-AAGAAATTGCCAAGAAAGACTGCCAGAGGTC TAAGAAAGGTTGC-3' $3^{\prime}$ and 5'-GCAACCTTTCTTAGACCTCTG GCAGTCTTTCTTGGCAATTTCTT-3'. The rpl3-H243A plasmid was then transformed into WT BY4742 using the LiOAc-PEGssDNA yeast transformation method (Gietz and Woods 2002). Subsequently, a CORE cassette was transformed into the WT cells at the endogenous RPL3 $\mathrm{H} 243$ position using a PCR product amplified by primers $5^{\prime}$-TACCCACAGATGGGGTACTAAGAAATT GCCAAGAAAGACTGAGCTCGTTTTCGACACTGG-3' and $5^{\prime}$ ATGCCAAGCACCAATACAAGCAACCTTTCTTAGACCTCTGT CCTTACCATTAAG TTGATC- $3^{\prime}$, according to the delitto perfecto method (Stuckey and Storici 2013). Next, a PCR product containing the H243A mutation was synthesized using Phusion High-Fidelity PCR Master Mix with HF buffer (NEB), primers $5^{\prime}$-GACTGTT GCTGTCGACAGC-3' and 5'-GTTCTGTCGAAGCTGGTAGC-3', and the pUG35 rpl3-H243A plasmid as a template. Finally, the PCR product was transformed into the cells carrying the CORE cassette at the endogenous RPL $3 \mathrm{H} 243$ locus. Cells were plated overnight on YPD and then replica-plated the next day to 5-fluoroorotic acid to select for removal of the pUG35 rpl3-H243A plasmid and replacement of the CORE cassette with the mutated H243A genomic sequence. The H243A mutation was confirmed by Sanger sequencing using primers $5^{\prime}$-CCAAATACTCTGCCAAGTACGC-3' and 5'-TCGAAGCTGGTAGCACCG-3' (Laragen Inc.). The open reading frame of HPM1 was cloned into the pET100/D-TOPO E. coli expression vector (Invitrogen), expressed and purified as described in Al-Hadid et al. (2014).

\section{In vivo radiolabeling with [methyl- ${ }^{3} \mathrm{H}$ ]AdoMet}

Overnight cultures of WT, $h p m 1 \Delta$, and $r p l 3-H 243 A$ cells were diluted in fresh YPD media to an $\mathrm{OD}_{600 \mathrm{~nm}}$ of 0.1 and grown at $30^{\circ} \mathrm{C}$ in a rotary shaker until the $\mathrm{OD}_{600} \mathrm{~nm}$ reached $0.8-1.0$. Seven $\mathrm{OD}_{600 \mathrm{~nm}}$ 
units from each culture were harvested by centrifugation at $5000 \mathrm{~g}$ for $5 \mathrm{~min}$ at room temperature and the cells were washed three times with water. Cells were then resuspended in $848 \mu \mathrm{L}$ of YPD and 152 $\mu \mathrm{L}$ of $S$-adenosyl- $\left[\right.$ methyl- $\left.{ }^{3} \mathrm{H}\right]$-L-methionine ([methyl- $\left.{ }^{3} \mathrm{H}\right]$ AdoMet; PerkinElmer; $83.3 \mathrm{Ci} / \mathrm{mmol}$; $0.55 \mathrm{mCi} / \mathrm{mL}$ in $10 \mathrm{mM} \mathrm{H}_{2} \mathrm{SO}_{4}$ :ethanol 9:1) and incubated at $30^{\circ} \mathrm{C}$ in a rotary shaker for $30 \mathrm{~min}$. Cells were then harvested by centrifugation and washed several times with water.

\section{Spheroplast preparation and subcellular fractionation}

Spheroplasts of WT, $h p m 1 \Delta$, and $r p l 3-H 243 A$ cells were prepared according to the protocol of Rieder and Emr (2001). Seven $\mathrm{OD}_{600 \mathrm{~nm}}$ units of cells were resuspended in $1 \mathrm{~mL}$ of $0.1 \mathrm{M}$ Tris sulfate, $10 \mathrm{mM}$ DTT, pH 9.4 and incubated at room temperature for $10 \mathrm{~min}$. Cells were centrifuged at $4000 \mathrm{~g}$ for $5 \mathrm{~min}$ and the supernatant aspirated. Cells were resuspended in $250 \mu \mathrm{L}$ of spheroplast medium A (SMA; $0.17 \%$ yeast nitrogen base without amino acids and ammonium sulfate [BD Biosciences], 2\% dextrose [Fisher; D16-1], 0.08\% complete supplement mixture [MP Biomedicals; 114500012], $1 \mathrm{M}$ sorbitol, and $20 \mathrm{mM}$ Tris-Cl, pH 7.5). Zymolyase 20T (Seikagaku Biobusiness) was added at a concentration of $1 \mathrm{mg} / 7 \mathrm{OD}_{600 \mathrm{~nm}}$ units and incubated in a $30^{\circ} \mathrm{C}$ rotary shaker for $30 \mathrm{~min}$. Spheroplasts were centrifuged at $1500 \mathrm{~g}$ for $5 \mathrm{~min}$ at room temperature and resuspended in $0.7 \mathrm{~mL}$ spheroplast recovery medium B (SMA without Tris-Cl). Spheroplasts were centrifuged again, the supernatant aspirated, and cells placed on ice until ready for lysis. For the in vitro methylation assay, spheroplasts were resuspended in buffer A (20 mM Tris base, $15 \mathrm{mM}$ magnesium acetate, $60 \mathrm{mM} \mathrm{KCl}, 1 \mathrm{mM}$ DTT, $1 \mathrm{mM}$ PMSF, Roche protease inhibitor cocktail [Roche; 11697498001], adjusted to pH 7.5 with $\mathrm{HCl}$ ) to a concentration of $15 \mathrm{OD}_{600} \mathrm{~nm}$ units/mL. For in vivo methylated cells, spheroplasts were resuspended in HEPES/KAc lysis buffer (20 mM HEPES, $50 \mathrm{mM}$ potassium acetate, $200 \mathrm{mM}$ sorbitol, $1 \mathrm{mM}$ EDTA, Roche protease inhibitor cocktail, $1 \mathrm{mM}$ PMSF, adjusted to $\mathrm{pH} 6.8$ with $\mathrm{KOH}$ ) to a concentration of $15 \mathrm{OD}_{600 \mathrm{~nm}}$ units/mL. Spheroplasts were lysed 10-20 times with a Dounce homogenizer. Lysates were transferred to prechilled tubes and centrifuged at $300 \mathrm{~g}$ for $5 \mathrm{~min}$ at $4^{\circ} \mathrm{C}$. The supernatant was transferred to a fresh tube and centrifuged at $13,000 \mathrm{~g}$ for 10 min. Supernatant $\left(S^{13,000}\right)$ was transferred to another tube. The pellet after the $13,000 \mathrm{~g}$ spin $\left(\mathrm{P}^{13,000}\right)$ is nuclei enriched. $\mathrm{P}^{13,000}$ was resuspended in $50 \mu \mathrm{L}$ of HEPES/KAc lysis buffer and placed on ice. $S^{13,000}$ was centrifuged at 20,000 $g$ for $10 \mathrm{~min}$ and the resulting supernatant (post-mitochondrial cell extract) was transferred to a fresh tube and placed on ice. Protein concentration was determined using the Lowry method after protein precipitation with $10 \%$ trichloroacetic acid.

\section{ACKNOWLEDGMENTS}

We thank Dr. William Munroe at UCLA for his assistance with the polysome profile analysis, Drs. Ming Du and David Bedwell at the University of Alabama for providing the stop codon readthrough and amino acid misincorporation plasmids, and finally Jonathan Dinman at the University of Maryland for providing the frameshifting plasmids. This work was supported by National Institutes of Health grants GM026020 (to S.G.C.) and GM061518 (to G.C.).
K.R. was supported by a Ruth L. Kirschstein National Research Service award GM007185.

Received September 23, 2015; accepted December 14, 2015.

\section{REFERENCES}

Al-Hadid Q, Roy K, Munroe W, Dzialo MC, Chanfreau GF, Clarke SG. 2014. Histidine methylation of yeast ribosomal protein Rpl3p is required for proper $60 \mathrm{~S}$ subunit assembly. Mol Cell Biol 34: 2903-2916.

Arnold RJ, Reilly JP. 1999. Observation of Escherichia coli ribosomal proteins and their posttranslational modifications by mass spectrometry. Anal Biochem 269: 105-112.

Baudin-Baillieu A, Fabret C, Liang XH, Piekna-Przybylska D, Fournier MJ, Rousset JP. 2009. Nucleotide modifications in three functionally important regions of the Saccharomyces cerevisiae ribosome affect translation accuracy. Nucleic Acids Res 37: $7665-7677$.

Ben-Shem A, Garreau de Loubresse N, Melnikov S, Jenner L, Yusupova G, Yusupov M. 2011. The structure of the eukaryotic ribosome at 3.0 A resolution. Science 334: 1524-1529.

Chang FN. 1978. Temperature-dependent variation in the extent of methylation of ribosomal proteins L7 and L12 in Escherichia coli. J Bacteriol 135: 1165-1166.

Chern MK, Chang KN, Liu LF, Tam TCS, Liu YC, Liang YL, Tam MF. 2002. Yeast ribosomal protein L12 is a substrate of protein-arginine methyltransferase 2. J Biol Chem 277: 15345-15353.

Clarke SG. 2013. Protein methylation at the surface and buried deep: thinking outside the histone box. Trends Biochem Sci 38: 243-252.

Cloutier P, Lavallée-Adam M, Faubert D, Blanchette M, Coulombe B. 2013. A newly uncovered group of distantly related lysine methyltransferases preferentially interact with molecular chaperones to regulate their activity. PLoS Genet 9: e1003210.

Dognin MJ, Wittmann-Liebold B. 1980. Identification of methylated amino acids during sequence analysis. Application to the Escherichia coli ribosomal protein L11. Hoppe Seylers Z Physiol Chem 361: 1697-1705.

Dzialo MC, Travaglini KJ, Shen S, Roy K, Chanfreau GF, Loo JA, Clarke SG. 2014. Translational roles of elongation factor 2 protein lysine methylation. J Biol Chem 289: 30511-30524.

Gautier T, Bergès T, Tollervey D, Hurt E. 1997. Nucleolar KKE/D repeat proteins Nop56p and Nop58p interact with Nop1p and are required for ribosome biogenesis. Mol Cell Biol 17: 7088-7098.

Gietz RD, Woods RA. 2002. Transformation of yeast by lithium acetate/ single-stranded carrier DNA/polyethylene glycol method. Methods Enzymol 350: 87-96.

Gottschling H, Freese E. 1962. A tritium isotope effect on ion exchange chromatography. Nature 196: 829-831.

Grandi P, Rybin V, Bassler J, Petfalski E, Strauss D, Marzioch M, Schäfer T, Kuster B, Tschochner H, Tollervey D, et al. 2002. $90 \mathrm{~S}$ pre-ribosomes include the $35 \mathrm{~S}$ pre-rRNA, the U3 snoRNP, and $40 \mathrm{~S}$ subunit processing factors but predominantly lack $60 \mathrm{~S}$ synthesis factors. Mol Cell 10: 105-115.

Harger JW, Dinman JD. 2003. An in vivo dual-luciferase assay system for studying translational recoding in the yeast Saccharomyces cerevisiae. RNA 9: 1019-1024.

Henras AK, Plisson-Chastang C, O’Donohue M-F, Chakraborty A, Gleizes P-E. 2015. An overview of pre-ribosomal RNA processing in eukaryotes. Wiley Interdiscip Rev RNA 6: 225-242.

Hori H. 2014. Methylated nucleosides in tRNA and tRNA methyltransferases. Front Genet 5: 144.

Johansson MJO, Byström AS. 2005. Transfer RNA modifications and modifying enzymes in Saccharomyces cerevisiae. Top Curr Genet 12: $87-120$.

Keeling KM, Lanier J, Du M, Salas-Marco J, Gao L, Kaenjak-Angeletti A, Bedwell DM. 2004. Leaky termination at premature stop codons 
antagonizes nonsense-mediated mRNA decay in S. cerevisiae. RNA 10: 691-703.

Lee S-W, Berger SJ, Martinović S, Pasa-Tolić L, Anderson GA, Shen Y, Zhao R, Smith RD. 2002. Direct mass spectrometric analysis of intact proteins of the yeast large ribosomal subunit using capillary LC/FTICR. Proc Natl Acad Sci 99: 5942-5947.

Lhoest J, Colson C. 1981. Cold-sensitive ribosome assembly in an Escherichia coli mutant lacking a single methyl group in ribosomal protein L3. Eur J Biochem 121: 33-37.

Lhoest J, Lobet Y, Costers E, Colson C. 1984. Methylated proteins and amino acids in the ribosomes of Saccharomyces cerevisiae. Eur J Biochem 141: 585-590.

Lipson RS, Webb KJ, Clarke SG. 2010. Rmt1 catalyzes zinc-finger independent arginine methylation of ribosomal protein Rps2 in Saccharomyces cerevisiae. Biochem Biophys Res Commun 391: $1658-1662$.

Meskauskas A, Dinman JD. 2010. A molecular clamp ensures allosteric coordination of peptidyltransfer and ligand binding to the ribosomal A-site. Nucleic Acids Res 38: 7800-7813.

Meskauskas A, Harger JW, Jacobs KLM, Dinman JD. 2003. Decreased peptidyltransferase activity correlates with increased programmed -1 ribosomal frameshifting and viral maintenance defects in the yeast Saccharomyces cerevisiae. RNA 9: 982-992.

Meskauskas A, Petrov AN, Dinman JD. 2005. Identification of functionally important amino acids of ribosomal protein L3 by saturation mutagenesis. Mol Cell Biol 25: 10863-10874.

Muranova TA, Muranov AV, Markova LF, Ovchinnikov YA. 1978. The primary structure of ribosomal protein L3 from Escherichia coli 70S ribosomes. FEBS Lett 96: 301-305.

Nesterchuk MV, Sergiev PV, Dontsova OA. 2011. Posttranslational modifications of ribosomal proteins in Escherichia coli. Acta Naturae 3: 22-33.

Odintsova TI, Müller EC, Ivanov AV, Egorov TA, Bienert R, Vladimirov SN, Kostka S, Otto A, Wittmann-Liebold B, Karpova GG. 2003. Characterization and analysis of posttranslational modifications of the human large cytoplasmic ribosomal subunit proteins by mass spectrometry and Edman sequencing. J Protein Chem 22: 249-258.

Oeffinger M, Zenklusen D, Ferguson A, Wei KE, El Hage A, Tollervey D, Chait BT, Singer RH, Rout MP. 2009. Rrp17p is a eukaryotic exonuclease required for $5^{\prime}$ end processing of Pre-60S ribosomal RNA. Mol Cell 36: 768-781.

Peltz SW, Hammell AB, Cui Y, Yasenchak J, Puljanowski L, Dinman JD. 1999. Ribosomal protein L3 mutants alter translational fidelity and promote rapid loss of the yeast killer virus. Mol Cell Biol 19: 384-391.

Piekna-Przybylska DD, Decatur WA, Fournier MJ. 2008. The 3D rRNA modification maps database: with interactive tools for ribosome analysis. Nucleic Acids Res 36: D178-D183.

Polevoda B, Sherman F. 2007. Methylation of proteins involved in translation. Mol Microbiol 65: 590-606.

Porras-Yakushi TR, Whitelegge JP, Miranda TB, Clarke S. 2005. A novel SET domain methyltransferase modifies ribosomal protein Rpl23ab in yeast. J Biol Chem 280: 34590-34598.
Ren J, Wang Y, Liang Y, Zhang Y, Bao S, Xu Z. 2010. Methylation of ribosomal protein S10 by protein-arginine methyltransferase $5 \mathrm{regu}-$ lates ribosome biogenesis. J Biol Chem 285: 12695-12705.

Rieder SE, Emr SD. 2001. Isolation of subcellular fractions from the yeast Saccharomyces cerevisiae. Curr Protoc Cell Biol 8: 3.8.13.8.68.

Rosado IV, Kressler D, de la Cruz J. 2007. Functional analysis of Saccharomyces cerevisiae ribosomal protein Rpl3p in ribosome synthesis. Nucleic Acids Res 35: 4203-4213.

Salas-Marco J, Bedwell DM. 2005. Discrimination between defects in elongation fidelity and termination efficiency provides mechanistic insights into translational readthrough. J Mol Biol 348: 801-815.

Shin HS, Jang CY, Kim HD, Kim TS, Kim S, Kim J. 2009. Arginine methylation of ribosomal protein S3 affects ribosome assembly. Biochem Biophys Res Commun 385: 273-278.

Sivan G, Kedersha N, Elroy-Stein O. 2007. Ribosomal slowdown mediates translational arrest during cellular division. Mol Cell Biol 27: 6639-6646.

Stuckey S, Storici F. 2013. Gene knockouts, in vivo site-directed mutagenesis and other modifications using the Delitto Perfetto system in Saccharomyces cerevisiae. Methods Enzymol 533: 103-131.

van Beekvelt CA, de Graaff-Vincent M, Faber AW, van't Riet J, Venema J, Raué HA. 2001. All three functional domains of the large ribosomal subunit protein L25 are required for both early and late pre-rRNA processing steps in Saccharomyces cerevisiae. Nucleic Acids Res 29: 5001-5008.

Venema J, Tollervey D. 1996. RRP5 is required for formation of both $18 \mathrm{~S}$ and $5.8 \mathrm{~S}$ rRNA in yeast. EMBO J 15: 5701-5714.

Venema J, Tollervey D. 1999. Ribosome synthesis in Saccharomyces cerevisiae. Annu Rev Genet 33: 261-311.

Webb KJ, Zurita-Lopez CI, Al-Hadid Q, Laganowsky A, Young BD, Lipson RS, Souda P, Faull KF, Whitelegge JP, Clarke SG. 2010a. A novel 3-methylhistidine modification of yeast ribosomal protein Rpl3 is dependent upon the YIL110W methyltransferase. J Biol Chem 285: 37598-37606.

Webb KJ, Lipson RS, Al-Hadid Q, Whitelegge JP, Clarke SG. 2010b. Identification of protein $\mathrm{N}$-terminal methyltransferases in yeast and humans. Biochemistry 49: 5225-5235.

Webb KJ, Al-Hadid Q, Zurita-Lopez CI, Young BD, Lipson RS, Clarke SG. 2011. The ribosomal L1 protuberance in yeast is methylated on a lysine residue catalyzed by a seven- $\beta$-strand methyltransferase. J Biol Chem 286: 18405-18413.

Woolford JL, Baserga SJ. 2013. Ribosome biogenesis in the yeast Saccharomyces cerevisiae. Genetics 195: 643-681.

Young BD, Weiss DI, Zurita-Lopez CI, Webb KJ, Clarke SG, McBride AE. 2012. Identification of methylated proteins in the yeast small ribosomal subunit: a role for SPOUT methyltransferases in protein arginine methylation. Biochemistry 51: 5091-5104.

Yu Y, Ji H, Doudna JA, Leary JA. 2005. Mass spectrometric analysis of the human 40S ribosomal subunit: native and HCV IRES-bound complexes. Protein Sci 14: 1438-1446. 

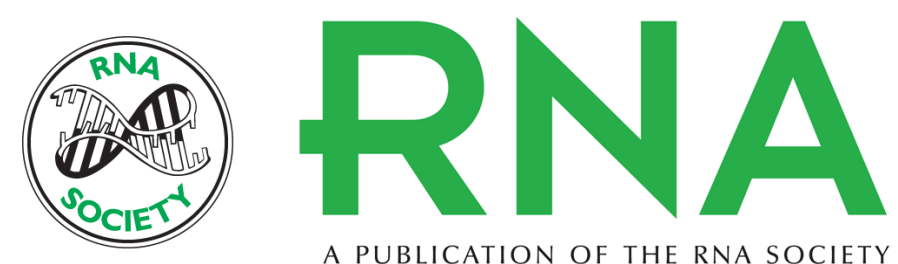

A PUBLICATION OF THE RNA SOCIETY

\section{Methylation of yeast ribosomal protein Rpl3 promotes translational elongation fidelity}

Qais Al-Hadid, Kevin Roy, Guillaume Chanfreau, et al.

RNA 2016 22: 489-498 originally published online January 29, 2016

Access the most recent version at doi:10.1261/rna.054569.115
References This article cites 50 articles, 18 of which can be accessed free at: http://rnajournal.cshlp.org/content/22/4/489.full.html\#ref-list-1
Creative This article is distributed exclusively by the RNA Society for the first 12 months after the Commons full-issue publication date (see http://rnajournal.cshlp.org/site/misc/terms.xhtml). After 12 License months, it is available under a Creative Commons License (Attribution-NonCommercial 4.0 International), as described at http://creativecommons.org/licenses/by-nc/4.0/.

Email Alerting Receive free email alerts when new articles cite this article - sign up in the box at the Service top right corner of the article or click here. 